\title{
ACUPUNCTURE AND BEE VENOM THERAPY IN THE CHRONIC LOW BACK PAIN: A SHORT REVIEW
}

\author{
Beatrice Lucache*, Elena Albu and Ostin C. Mungiu \\ Centre for the Study and Therapy of Pain, Faculty of Medicine \\ "Grigore T. Popa" University of Medicine and Pharmacy \\ 16 Universităţii Str., 700115 - Iași, Romania \\ *E-mail: beatrice.lucache@gmail.com, elenaalbu@yahoo.com, ocmungiu@yahoo.com
}

\begin{abstract}
Background: Many pharmacological and nonpharmacologic therapies are available for the treatment of the low back pain (LBP). Despite medications are the most frequently prescribed therapy for LBP, the use of various complementary medicine techniques is increasing. The paper summarizes the latest evidence on the treatment of musculoskeletal conditions (with special focus on chronic LBP) by using acupuncture and bee venom therapy (BVT).

Methodology: The overview is based on English-language studies and articles found by searches of Medline over more than last 10 years. All the paper found that refer to LBP and at least one of the analyzed therapies have been taken into account.

Results: The mechanism of action, efficiency, harms, toxicity and contraindications are highlighted for the two therapies. The literature on these topics is extensive and controversial. There are trials in favour of using the two complementary therapies for rheumatic diseases, as well trials arguing that the observed analgesic effects are related to a strong placebo response.

Conclusions: The choice of using acupuncture and BVT for chronic LBP depends on various factors related to patient features and preferences, but nowadays an increased attention and support has to be noted.
\end{abstract}

Key words: Chronic low back pain, Acupuncture, Bee venom therapy.

\section{Introduction}

The chronic low back pain (CLBP) represents a major health problem having high social impact, characterized by important costs and economics losses, but also an important suffering source in individual plan (Chenot et al., 2007). In the industrialized countries, back pain affects between $15 \%$ and $30 \%$ of the population and generates high costs (Chou and Huffman, 2007). There are many therapeutic treatments available, but is unclear from the literature which intervention is the most effective in treating nonspecific LBP, which represent about $90 \%$ from total cases of LBP (Hutchinson et al., 2012).

\section{Acupuncture}

Acupuncture has been successfully incorporated into medical or allied health practices in Western countries. As a means of treatment, in China has been in use for about 3000 years. In its classical definition, acupuncture prevents and treats disease by the painless insertion of needles at certain key points in the patient's body. Within the context of acupuncture, it is accepted that these points are part of a system and are connected by channels, or meridians (Motl, 2002).

Acupuncture focuses on a holistic, energy-based approach to the patient, rather than a disease-oriented diagnostic and treatment model. When integrated with Western-based Medicine, acupuncture is a very practical discipline, with a wide range of clinical applications, from pain management problems to functional disturbances (Fargas-Babjak, 2004). Acupuncture is thought to have a general homeostatic effect, facilitating the activity of the body's natural healing mechanisms, thus returning the body to its normal functional state. When used for the therapeutic purpose, it modulates the abnormal activity of the nervous system and/or of its associated effectors, the endocrine and exocrine systems.

Even though considerable scientific bases have been proven, the mechanisms of action of acupuncture in terms of Western scientific principles have not been completely revealed, including in the case of chronic low back pain (Ammendolia et al., 2008). There are used two most common models for the acupuncture mechanisms of action: the standard neurophysio-logical model and the gate-control model. In the first one, the analgesic effects of acupuncture are at least partially explained by the actions of the opioid peptides ( $\beta$-endorphin, enkephalin, endomorphin, and dynorphin) (Chou et al, 2012; Han, 2003) as well as hormones such as adrenocortico-trophin (ACTH) and cortisone (Carlsson, 2002), which are released during acupuncture.

The gate-control theory of pain introduced first by Melzack and Wall (1965) explains the observed pain-relief resulting from stimulation of somatic afferent nerves. The wide-dynamic-range neurones, located in the dorsal horn, receive input from both nociceptive and non-nociceptive afferents and are inhibited by interneurones that are excited by non-noxious inputs in the same segment. This mechanism only explains pain relief during stimulation and it does not explain why pain inhibition may outlast the duration of stimulation by hours or even days (Carlsson, 2002).

In the segmental acupuncture (needles are inserted within the segment of pain) the physiology is related to the spinal gate-control mechanism (involving enkephalin and dynorphin). In the extra-segmental acupuncture the hypothalamus and the pituitary gland may be activated periaqueductal grey, and the descending pain relieving system (involving endorphins, serotonin and noradrenaline) (Price and Meyer, 1995) resulting in a broad spectrum of systemic effects. There are also claimed to be involved the diffuse noxious inhibitory control (Bing et al., 1990) and/or the activation of the hypothalamic pituitary-adrenal (HPA)-axis with increased levels (in the blood) of $\beta$-endorphin and ACTH/ cortisone (Pomeranz, 2000; Han, 1987).

More recently the promising new researches utilizing functional magnetic resonance imaging seem to indicate the connection of the autonomic nervous system with acupuncture through the inflammatory reflex, which regulates the immune system in the organism (Lin and Chen, 2008). 


\section{http://dx.doi.org/10.4314/ajtcam.v12i1.11}

The most successful applications of acupuncture are reported in acute and chronic musculoskeletal pain. Pain problems responsive to acupuncture include: acute sprains and strains, pain from nerve entrapment, repetitive strain disorders (e.g. carpal tunnel syndrome, tennis elbow, plantar fasciitis, Achilles tendonitis), myofascial pain patterns (e.g. tempero-mandibular joint pain, muscle tension headaches, shoulder pain, neck and back pain), arthralgias (particularly osteoarthritic in nature), degenerative disc disease with or without radicular pain, pain following surgical intervention (both musculo-skeletal and visceral), post herpetic neuralgia, peripheral neuropathic pain and miscellaneous headaches (FargasBabjak, 2004).

In CLBP, a report done by Staud (2007) concludes that is strong evidence favouring the acupuncture short-term analgesic effectiveness compared with most other therapies. The data analysed by Furlan et al. (2008) suggest also that acupuncture may be useful adjunct to other therapies for CLBP. More, the acupuncture effects on CLBP pain seem to last for up to 2 years (Thomas et al, 2005). On the opposite, in the treatment of acute low back pain, acupuncture data are considered still sparse and inconclusive (Staud, 2007).

\section{Acupuncture toxicity and harms}

Acupuncture is generally considered safe, harmless and non-toxic. But, as the effectiveness of acupuncture remains still controversial because the mechanisms of action are not completely revealed, the lack of side effects is another issue for disputes.

There are several methods to do acupuncture. The standard or traditional acupuncture means the insertion of acupuncture needles into the meridian points, while the patient is supposed to experience a sensation known as De-qi. Starting from this, new forms of acupuncture have been developed, such as ear (auricular) acupuncture, head (scalp) acupuncture, hand acupuncture and foot acupuncture (Lao, 1996). Acupuncture commonly includes manual stimulation of the needles, but various adjuncts are often used, including electrical acupuncture (in which an electrical stimulator is connected to the acupuncture needle), injection acupuncture (herbal extracts injected into acupuncture points), heat lamps, and acupuncture with moxibustion (the moxa herb, Artemisia vulgaris, is burned at the end of the needle) (Yanashita et al., 2000). So, the possible acupuncture toxicity depends on the applied method. The techniques using dry needles are considered without toxicity, while the wet (injection) acupuncture assumes the toxicity of the injected substance.

Acupuncture, as any intervention on the body, cannot be harmless. Impressive number of studies, case reports, surveys and prospective studies has been dedicated to the safety of the acupuncture treatments. There are two kinds of approaches: the believers in acupuncture, which consider this treatment near "harmless" and the sceptical ones that try to definitive demolish the "harmless myth". Literature brought evidence for all kind of adverse effects, from the less significant up to severe adverse reactions, even death.

The most recorded systemic reactions in individual patients were: tiredness, drowsiness, aggravation of pre-existing symptoms, itching in the punctured regions, dizziness or vertigo, feeling of faintness or nausea during treatment, vomiting, headache and chest pain. Low incidence rate of the recorded local reactions (minor bleeding on withdrawal of the needle, pain on insertion of the needle, petechia or ecchymosis; pain or ache in the punctured region after the treatment; subcutaneous haematoma; pain or discomfort in the punctured region during the needle retention) has been found by Yanashita et al. (2000).

Types of complications included infections (primarily hepatitis from a few practitioners), and organ, tissue, and nerve injury (Lao et al., 2003). Serious adverse effects including pneumothorax, spinal lesions, and hepatitis B transmission have been reported in the literature, but these are rare and generally associated with poorly trained unlicensed acupuncturists (Vickers et al., 2002).

In a comprehensive study, Ammendolia (2008) identified twelve trials reported complications or side effects related to acupuncture for CLBP. These all were of a minor nature and most involved local bleeding or hematoma, the reported rate being between 6 and $8 \%$ of the patients.

Among the main withstanders of the myth "acupuncture is harmless", Erik Ernst (2010) done a kind of systematically review of previous systematically reviews, and found up to 86 instances of deaths after acupuncture. The most frequent causes of death were pneumothorax, various types of (mostly bacterial) infections and accidental puncture of organs like heart, liver or large blood vessel, as well of the central nervous system. The majority of cases happened in China or other Asian countries, at least some of these might have been avoided by better training in anatomy and infection control.

Among all acupuncture associated complications, transmission of pathogenic microorganisms is one of the most important (Woo et al., 2010). Few decades ago, the majority of the infections associated with acupuncture were sporadic cases of pyogenic bacterial infections, mainly due to Staphylococcus aureus, as well as apparitions of hepatitis B virus infections. Although, the use of single-use or single-person needles led to a lower incidence of blood borne viruses transmitted by acupuncture. Nevertheless, in 2009, there were first reports of methicillin resistant Staphylococcus aureus transmitted by acupuncture (Woo, 2011).

Acupuncture is not recommended for patients who have haemophilia, bleeding disorders, and septicaemia, or those with cellulitis, local skin infections, or loss of skin integrity from burns or ulcerations in the case of needling. Extra care should be taken for individuals who are pregnant, have metal allergies, or are taking anticoagulant drugs (Sierpina and Frenkel, 2005).

\section{Bee venom therapy}

Among the bee products, the bee venom has the largest therapeutic area. The bee venom therapy (BVT) gained an increased interest in the last decades. Bee venom is administered in the form of a direct bee sting or else by injection of a venom extract, and requires the expertise of a qualified health care professional. Bee venom has been reported to have a central analgesic mechanism and a peripheral analgesic mechanism along with a peripheral analgesic action from an anti-inflammatory effect through specific mechanisms (Shin et al., 2012).

When the bee venom is injected into acupuncture points (acupoint), the method calls bee venom acupuncture (BVA), which was proven successful for producing a strong therapeutic effect as compared to injection in the non-acupoint areas (Kwon et al., 2001). In BVA the mechanical effect from the stimulation of the acupuncture point has a supplementary action beside the pharmacological effect from the bioactive bee venom compounds (Lee et al., 2005). On the other side, compared to classical acupuncture treatment, BVA is considered superior due to the additional pharmacological effect of bee venom (Lee at al., 2008).

\section{Bee venom composition and proprieties}

Bee venom contains more than 18 different compounds that all possess pharmaceutical properties. Bee venom is a complex mixture of proteins, peptides and low molecular components. The composition of fresh and dried bee venom differs mainly in regards to the volatile components, but the overall biological activity remains similar (Bogdanov, 2014). 
http://dx.doi.org/10.4314/ajtcam.v12i1.11

The main components of the bee venom are the enzymes (proteins catalyzing specific reactions, as Phospholipase A2, Phospholipase B and Hyalyronidase etc.) and peptides (Melittin, Apamine, MCD peptide, Procamine etc.). Beside these, bee venom contains smaller quantities of low molecular compounds that are different in nature: amino acids, catecholamines (Histamine, Dopamine and Noradrenalin), sugars and minerals $(\mathrm{Ca}, \mathrm{Mg}, \mathrm{P})$.

The analgesic, anti-inflammatory, anti-cancer, and immuno-stimulant effects have been proven in numerous studies with animals (Huh et al., 2010; Kim et al., 2008; and others). Several studies have reported also radio-protective and anti-mutagenic effects of bee venom, both in vitro and in vivo (Hassanein and Hegab, 2010).

Bee Venom Therapy stimulates the immune system through the hypothalamus, pituitary and adrenal glands. As consequence, the body produces its own healing agents. The healing mechanism is not completely revealed, but ongoing research has identified a number of naturally occurring chemical compounds, which appear to work together in the body. One possible explanation of the anti-inflammatory effects is the contribution to the production of endogenous plasma cortisol in the human body. Unlike the synthetic cortisone this has no side effects and plays an important role in the regulation of lymphocyte numbers as well exert their effect on stressful task performance via modulation of the amygdala (Sarria, 2012; Kwon et al., 2003).

Melittin, a polypeptide occupying over $50 \%$ of the whole honeybee venom, plays a central role in production of local inflammation, nociception and hyperalgesia or allodynia (Chen, 2006). Melittin suppress inflammation by inhibiting enzymatic activity, an enzyme that is abundantly released in severe inflammatory disorders and causes tissue and organ degradation (Mihelich and Schevitz, 1999).

Amongst the most important of the bee venom components seems to be the mast cell degranulating peptide (MCD) peptide, known also as Peptide-401. Banks (1976) demonstrates that MCD peptide is 100 times more potent than hydrocortisone in the rat paw edema test. It is also a better inhibitor than aspirin of the conversion of arachidonic acid to PE2.

Moreover, bee venom contains a variety of bioamines, such as apamin, dopamine (helps increasing motor activity), histamine, procamine, serotonin, which facilitate nerve transmission and healing in a variety of nerve disorders. Adolapin, also a neurotransmitter, is a strong antiinflammatory substance with analgesic activity as well. Apamin enhances long term synaptic transmission by significantly inhibiting the activity of C3 complement, and blocks calcium-dependent potassium channels. This gives bee venom the ability to travel along the neural pathways from the spine to various trigger points and injured areas to help repair nerve damage and restore mobility (Rakha, 2013).

Compound X, hyaluronidase, phospholipase A2, histamine, and MCD peptide, are also involved in the inflammatory response of venom, with softening of tissue and facilitation of flow of other beneficial substances to damaged areas of nervous tissue (Mraz, 2001).

The bee venom's anti-inflammatory effect is dependent on peripheral nerve integrity. Through a transection of the sciatic nerve, Kwon et al. (2006) showed that the anti-inflammatory effect completely disappears, being not a locally mediated anti-inflammatory effect.

In the last decades a lot of scientific articles brought sound evidence of the effectiveness of bee venom against arthritis and painful inflammatory conditions. BVT, due to the anti-inflammatory and analgesic effects, have been used for the treatment of different rheumatic diseases as low back pain, neck pain, disc pain, herniated lumbar pain, rheumatoid arthritis etc. The evidence of effectiveness of the BVA in the musculoskeletal pain management was analyzed and positive concluded by Lee et al. (2008) in a comprehensive review.

\section{Bee venom toxicity and allergic reactions}

Based upon all the literatures, there should be no strong objections to the use of bee venom. Actually, the great majority of authors who address themselves to the effects of apitherapy report little undesirable reactions, as long as the patient is not allergic to bee venom (Kim, 1997). Bee venom treatment did not affect motor activity, intestinal peristaltic function or gastric function. Additionally, bee venom did not alter blood pressure and heart rate in rats nor respiratory rates in rabbits (Kim et al., 2004)

Usually, bee venom is administered in the form of a direct bee sting or else by injection of a venom extract, and the treatment starts with testing the patient for allergy, which is known to occur in $1 \%$ of the general population (Mahmoud, 2012). A study done by Castro et al. (2005) confirmed that the administration of repeated injections of bee venom in a step-up dosage regime, in non-bee venom allergic patients with multiple sclerosis, had no serious adverse allergic reactions.

The bee venom is safe for human treatments, the median lethal dose (LD50) for an adult human being $2.8 \mathrm{mg}$ of venom per kg of body weight. Assuming a bee injects up to $0.3 \mathrm{mg}$ venom per sting, results that a person could resist normally to hundreds of bee stings, depending on its weight. Statistics shown most human deaths result from one or few bee stings due to allergic reactions, heart failure or suffocation from swelling around the neck or the mouth (Mahmoud, 2012).

In getting the desensitization, the venom immunotherapy may be responsible for local and systemic allergic reactions. Purified aqueous and purified aluminium hydroxide adsorbed extracts have the potential to reduce the incidence of venom immunotherapy side effects (Bilò et al., 2010).

Few reports have claim severe toxicities developing in response to bee venom therapy (Park et al., 2010; Mytas et al., 2009). Hemoconcentration can appear due to bee sting and might be related to the marked edema induced by the venom. Increase in various cytokines like interleukin (IL)-1 $\beta$, IL- 6 , tumour necrosis factor- $\alpha$, etc. have been reported after bee sting.

If just few stings are considered safe for non-allergic persons, mass inoculation of bee venom may induce acute renal failure, adult respiratory distress syndrome, liver injury, cardiac damage, pancreatitis, skin necrosis, shock hypertension, bleeding, thrombocytopenia, and rhabdomyolysis (Kluwer, 2009).

\section{Discussion}

The personal research done by authors has conclusions not far from the ones from literature. Regarding effectiveness, in a comparative study on the efficiency of acupuncture vs. diclofenac (Author, 2013), the authors found statistically significant different but moderate-better results in favour of the discussed complementary therapy. This is similar to Kaptchuk (2010) which found out that the recent scientific evidence for acupuncture and CLBP has not been as spectacular as the majority of acupuncture practitioners claim.

In the absence of health assurance reimbursements, the complementary therapies need more time consumption and are usually costlier than the simply medication. Between the explanations of the efficiency and attractiveness of the complementary and alternative could be, as Cherkin et al. (2001) mentioned, the longer time spent by patient (up to one hour) in a relaxed environment, the ongoing attention and the therapeutic touching. Also, acupuncture might enhance mental health independent on its effects on the physical health and BVT improves neurotransmission 
http://dx.doi.org/10.4314/ajtcam.v12i1.11

and facilitates repairing of tissue areas. The people that prefer this kind of treatment either are allergic to the drugs administration or pursue complementary benefic effects from the complementary therapies.

Regarding harms and toxicity, the complementary therapies are considered less invasive than long cures with medication. The main worry and caution is related to the bee venom toxicity that can be immediate or can manifest itself only weeks after the exposure. A case of hepatotoxicity at three weeks after BVT for was reported by Alqutub et al. (2011). The authors experienced a quite similar experience with a 36-year-old female that suffered a major unexpected anaphylactic shock in the sixth treatment session of BVT for CLBP.

\section{Conclusion}

The paper makes an up-to-date short review on the main stream literature found on Medline regarding acupuncture and BVT for the CLBP. Although the results of the studies and trials remain still controversial regarding the efficiency, although toxicity potential could be important for certain patients or harm potential exist because of untrained and inexperienced practitioners, both analyzed complementary therapies are taken into consideration as ones of the cost-efficient alternatives procedures in CLBP treatment. It is important to keep in mind that, these are not necessary a substitution variant for the pharmaceutical treatment and can be mixed with the last one in the patient benefit.

\section{References}

1. Alqutub, A.N., Masoodi, I., Alsayari, K., Alomair, A. (2011). Bee sting therapy-induced hepatotoxicity: A case report. World Journal of Hepatology. 3(10): 268-270.

2. Ammendolia, C., Furlan, AD, Imamura, M., Irvin, E, van Tulder M. (2008). Evidence-informed management of chronic low back pain with needle acupuncture. Elsevier. The Spine Journal. 8 160-172.

3. Authors. (2013). Pharmaceutical and acupuncture treatments comparison in the chronic low back pain. Medical-Surgical Journal. 117(2), Supl. 1, pp.62-66.

4. Banks, B. (1976). Possible Therapeutic Use of a Peptide from Bee Venom. Bulletin de L'Institut Pasteur. 74, p137-44.

5. Bilò, MB, Antonicelli, L., Bonifazi, F. (2010). Purified vs. nonpurified venom immunotherapy. Curr Opin Allergy Clin Immunol. 10(4): 3306.

6. Bing, Z., Villanueva, L., Le Bars, D. (1990). Acupuncture and diffuse noxious inhibitory controls: naloxone-reversible depression of activities of trigeminal convergent neurons. Neuroscience. 37(3):809-18.

7. Bogdanov, S. (2014). The Bee Venom Book. Bee Product Science. (http://www.bee-hexagon.net).

8. Carlsson, C. (2002). Acupuncture mechanisms for clinically relevant long-term effects - reconsideration and a hypothesis. Acupuncture in Medicine. 20(2-3):82-99.

9. Castro, H.J., Mendez-Lnocencio, J.I., Omidvar, B., Omidvar, J., Santilli, J., Nielsen, H.S. Jr, Pavot, A.P., Richert, J.R., Bellanti, J.A. (2005). A phase I study of the safety of honeybee venom extracts as a possible treatment for patients with progressive forms of MS. Allergy Asthma Proc. 26:470-476.

10. Chen, Y.N., Li, K.C., Li, Z., Shang, G.W., Liu, D.N., Lu, Z.M., Zhang, J.W., Ji, Y.H., Gao, G.D., Chen, J. (2006). Effects of bee venom peptidergic components on rat pain-related behaviors and inflammation. Neuroscience. 138(2):631-40.

11. Chenot, J.F., Becker, A., Leonhardt, C., Keller, S., Donner-Banzhoff, N., Baum, E., Pfingsten, M., Hildebrandt, J., Basler, H.D., Kochen, M.M. (2007). Use of complementary alternative medicine for low back pain consulting in general practice: a cohort study. BMC Complementary and Alternative Medicine. 7(42).

12. Cherkin, D.C., Eisenberg, D., Sherman, K.J., Barlow, W., Kaptchuk, T.J., Street, J., Deyo, R.A. (2001). Randomized trial comparing traditional Chinese medical acupuncture, therapeutic massage, and self-care education for chronic low back pain. Arch Intern Med. 161(8):1081-8.

13. Chou, L-W., Kao, M-J., Lin, J-G. (2012). Probable Mechanisms of Needling Therapies for Myofascial Pain Control. Evidence-Based Complementary and Alternative Medicine. 705327:1-11.

14. Chou, R., Huffman, LH. (2007). Nonpharmacologic therapies for acute and chronic low back pain: a review of the evidence for an American Pain Society/American College of Physicians clinical practice guideline. Ann Intern Med. 147(7):492-504.

15. Ernst E. (2010). Deaths after acupuncture: a systematic review. International Journal of Risk \& Safety in Medicine. 22:131-136.

16. Fargas-Babjak, A. (2004). Acupuncture Division: Application of contemporary medical acupuncture as a neuromodulation technique in pain management. Orthopaedic Division Review. (www.orthodiv.org).

17. Furlan, A.D., van Tulder, M.W., Cherkin, D., Tsukayama, H., Lao, L., Koes, B.W., Berman, B.M. (2008). Acupuncture and dry-needling for low back pain (Review). The Cochrane Collaboration. Published by JohnWiley \& Sons, Ltd. The Cochrane Library 2008 , Issue 4.

18. Han, JS. (2003). Acupuncture: neuropeptide release produced by electrical stimulation of different frequencies. Trends Neurosci. 26:17-22.

19. Han, JS. (1987).The neurochemical basis of pain relief by acupuncture. A collection of Papers 1973-1987. Beijing.

20. Hassanein, N., Hegab, A. (2010). Bee Venom - Lead Acetate Toxicity Interaction, Australian Journal of Basic and Applied Sciences. 4(8): 2206-2221.

21. Huh, J.E., Baek, Y.H., Lee, M.H., Choi, D.Y., Park, D.S, L,ee, J.D. (2010). Bee venom inhibits tumor angiogenesis and metastasis by inhibiting tyrosine phosphorylation of VEGFR-2 in LLC-tumor-bearing mice. Cancer Lett. 292(1):98-110.

22. Hutchinson, A.J., Ball, S., Andrews, J.C., Jones, G.G. (2012).The effectiveness of acupuncture in treating chronic non-specific low back pain: a systematic review of the literature. J Orthop Surg Res. 2012 Oct 30; 7:36.

23. Kaptchuk, T.J., Chen, K.J., Song, J. (2010). Recent clinical trials of acupuncture in the West: responses from the practitioners. Chin J Integr Med. 16(3): 197-203.

24. Kim, C.M-H. (1997). Apitherapy (Bee Venom Therapy), in Potentiating Health and the Crisis of the Immune System. Plenum Press, New York. Chapter 24: 243-270.

25. Kim, H.W., Kwon, Y.B., Ham, T.W., Roh, D.H., Yoon, S.Y., Kang, S.Y., Yang, I.S., Han, H.J., Lee, H.J., Beitz, A.J., Lee, J.H. (2004). General pharmacological profiles of bee venom and its water soluble fractions in rodent models. J Vet Sci. 5(4):309-18.

26. Kim, K.W., Shin, Y.S., Kim, K.S., Chang, Y.C., Park, K.K., Park, J.B., Choe, J.Y., Lee, K.G., Kang, M.S., Park, Y.G., Kim, C.H. (2008). Suppressive effects of bee venom on the immune responses in collagen-induced arthritis in rats. Phytomedicine 15. (12):1099-107.

27. Kluwer, W. (2009). Health: Bee Venom - Print Version, (www.drugs.com/npp/bee-venom.html?printable=1) 
http://dx.doi.org/10.4314/ajtcam.v12i1.11

28. Kwon, Y.B., Kim, H.W., Ham, T.W., Yoon, S.Y., Roh, D.H., Han, H.J., Beitz, A.J., Yang, I.S., Lee, J.H. (2003).The anti-inflammatory effect of bee venom stimulation in a mouse air pouch model is mediated by adrenal medullar activity. J Neuroendocrinol . 15(1):93-96.

29. Kwon, Y.B., Lee, JH., Han, HJ., Mar, WC., Beitz, AJ., Lee, HJ. (2001). "Bee venom injection into an acupuncture point reduces arthritis associated edema and nociceptive responses". Pain. 90: 271-280.

30. Kwon, Y.B., Yoon, S.Y., Kim, H.W., Roh, D.H., Kang, S.Y., Ryu, Y.H., Choi, S.M., Han, H.J., Lee, H.J., Kim, K.W., Beitz, A.J., Lee, J.H. (2006). Substantial role of locus coeruleus-noradrenergic activation and capsaicininsensitive primary afferent fibers in bee venom's antiinflammatory effect. Neurosci Res. 55(2):197-203.

31. Lao, L., Hamilton, GR., Fu, J., Berman, BM. (2003). Is acupuncture safe? A systematic review of case reports. Alternative Therapies. 9(1):72-83.

32. Lao, L. (1996). Safety issues in acupuncture. J Altern Complement Med. 2(1):27-31.

33. Lee, JD., Park, HJ., Chae, Y., Lim, S. (2005). An overview of bee venom acupuncture in the treatment of arthritis. Evid Based Complement Altern Med. 2(1):79-84.

34. Lee, MS., Pittler, MH., Shin, BC., Kong, JC., Ernst, E. (2008). Bee venom acupuncture for musculoskeletal pain: a review. J Pain. 9(4):28997.

35. Lin, J-G., Chen, W-L. (2008). Acupuncture Analgesia: A Review of Its Mechanisms of Actions. The American Journal of Chinese Medicine. Vol. 36, No. 4, 635-645.

36. Mahmoud, A. (2012). Studies on Bee Venom and Its Medical Uses, International Journal of Advancements in Research \& Technology. $1(2): 1-15$.

37. Melzack, R., Wall, PD. (1965). Pain mechanisms: a new theory. Science. 150(3699):971-979.

38. Mihelich, ED., Schevitz, RW. (1999). Structure-based design of a new class of anti-inflammatory drugs: secretory phospholipase A(2) inhibitors, SPI. Biochim. Biophys. Acta. 1441: 223-228.

39. Motl, MJ. (2002). Acupuncture. Chapter 20, Handbook of Complementary and Alternative Therapies in Mental Health. Academic Press, Inc.

40. Mraz, C. (2001). Health and the Honeybee. Queen City Publishing, Burlington, VT.

41. Mytas, D.Z, Stougiannos, P.N., Zairis, M.N., Tsiaousis, G.Z., Foussas, S.G., Hahalis, G.N., Kounis, N.G., Pyrgakis, V.N. (2009). Acute anterior myocardial infarction after multiple bee stings. A case of Kounis syndrome. Int J Cardiol. 134: e129-e131.

42. Park, S., Chun, H.J., Keum, B., Seo, Y.S., Kim, Y.S., Jeen, Y.T., Lee, H.S., Um, S.H., Kim, C.D., Ryu, H.S. (2010). Anaphylactic shockinduced ischemic proctocolitis following bee stings: first case report. Endoscopy. 42 Suppl 2: E153-E154.

43. Pomeranz, B. (2000). Acupuncture Analgesia - Basic Research. Clinical Acupuncture: Scientific Basis. Springer-Verlag, Berlin. pp1-28.

44. Price, DD., Mayer, DJ. (1995). Evidence for endogenous opiate analgesic mechanisms triggered by somatosensory stimulation (including acupuncture) in humans. Pain Forum. 4(1):40-3.

45. Rakha, MK. (2013). Neuroprotective and Neurotherapeutic Effects of Bee Venom on Neurodegenerative Diseases. Front. Hum. Neurosci. Abstract. doi: 10.3389/conf. fnhum.2013.210.00057.

46. Sarria, M. (2012). Integrative bee therapy (BVT) - Apitherapy in Integrative Chi Kung, eBook First English Edition. (http://www.ichikung.com).

47. SHIN, B. C., KONG, J C.., PARK, T. Y., YANG, C. Y., KANG, K. W., CHOI, S. M. (2012). Bee venom acupuncture for chronic low back pain: A randomised, sham-controlled, triple-blind clinical trial, European Journal of Integrative Medicine. 4, e271-e280.

48. Sierpina, VS., Frenkel, MA. (2005). Acupuncture: a clinical review. South Med J 98:330-7.

49. Staud, R. (2007). Mechanisms of Acupuncture Analgesia: Effective Therapy for Musculoskeletal Pain?. Current Rheumatology Reports. 9:473-481.

50. Thomas, K.J., MacPherson, H., Ratcliffe, J., Thorpe, L., Brazier, J., Campbell, M., Fitter, M., Roman, M., Walters, S., Nicholl, J.P. (2005). Longer term clinical and economic benefits of offering acupuncture care to patients with chronic low back pain. Health Technol Assess, (32): iii-iv, ix-x, 1-109.

51. Vickers, A., Wilson, P., Kleijnen J. (2002). Acupuncture. Qual Saf Health Care. 11:92-97.

52. Woo, PCY., Lin, AWC., Lau, SKP. (2011). Acupuncture Transmitted Infections, Acupuncture - Clinical Practice. Particular Techniques and Special Issues. Prof. Marcelo Saad (Ed.). InTech. ISBN: 978-953-307-630-0.

53. Woo, P.C., Lin, A.W., Lau, S.K., Yuen, K.Y. (2010). Acupuncture transmitted infections. BMJ. Vol.340:1151-1152.

54. Yamashita, H., Tsukayama, H., Hori, N., Kimura, T., Tanno, Y. (2000). Incidence of adverse reactions associated with acupuncture. J Altern Complement Med. 6(4):34. 